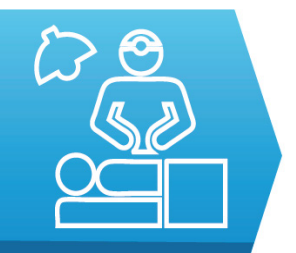

SURGERY

\title{
Laparoscopic sleeve gastrectomy as a primary bariatric procedure: postoperative outcomes
}

\author{
Flavius Mocian, Marius Coroș
}

Third Department of Surgery, George Emil Palade University of Medicine, Pharmacy, Science and Technology of Târgu Mureș, Târgu Mureș, Romania
DOI: $10.15386 / \mathrm{mpr}-1762$

Manuscript received: 05.06.2020

Accepted: 25.09.2020

Address for correspondence:

mocianfla@gmail.com

This work is licensed under a Creative Commons Attribution-NonCommercialNoDerivatives 4.0 International License

\begin{abstract}
Background and aims. Laparoscopic sleeve gastrectomy is at present the most popular bariatric procedure due to its significant effect on weight loss, resolution of comorbidities and improvement in the quality of life. However, there are not many studies showing its medium and long term efficacy and safety in Eastern Europe. We aim to report the outcomes of laparoscopic sleeve gastrectomy as a primary bariatric procedure from a single surgical department.
\end{abstract}

Methods. This is a retrospective analysis of obese patients who underwent laparoscopic sleeve gastrectomy between 2009 - 2016 in our department. Outcomes were analyzed in terms of weight loss, comorbidity resolution and quality of life changes.

Results. A number of 70 patients were included in the study, with a mean age of $47.6 \pm 9.8$ years. Mean initial Body Mass Index was $47.4 \pm 6.4 \mathrm{~kg} / \mathrm{m}^{2}$. The mean percentage of excess weight loss during follow-up was $78.2 \%$ at 12 months, $80.2 \%$, $76.7 \%, 74.7 \%, 72.8 \%, 73.3 \%, 74.1 \%, 67.2 \%, 64.07 \%, 69.7 \%$ at $24,36,48,60,72$, 84, 96, 108 and 120 months, respectively. Weight regain occurred in $61(87.1 \%)$ patients during the postoperative period. The mean weight regained was $8.1 \pm 4.8$ $\mathrm{kg}$ at 24 months postoperatively. Regarding the quality of life, $83 \%$ of patients declared an increase in self-esteem. Resolution of diabetes, arterial hypertension, and obstructive sleep apnea syndrome occurred in $80 \%, 42.5 \%$ and $70.1 \%$ of patients respectively.

Conclusions. Laparoscopic sleeve gastrectomy is an effective procedure, with good outcomes in the medium and long term, although a tendency for weight regain was noted after 2 years. Resolution of comorbidities is comparable with that reported in the literature, namely an improvement of the quality of life.

Keywords: laparoscopic sleeve gastrectomy, weight loss, quality of life, weight gain

\section{Background and aims}

In the $21^{\text {st }}$ century, obesity represents a public health problem, which is constantly increasing. Worldwide obesity has almost tripled since 1975 and it is estimated that approximately $13 \%$ of the adult population has a Body Mass Index (BMI) of $30 \mathrm{~kg} / \mathrm{m}^{2}$ or over [1]. Obesity is associated with comorbidities such as diabetes, high blood pressure, sleep apnea, gastroesophageal reflux disease, joint disease and others [2]. Nowadays, this is one of the main public health issues, due to associated comorbidities affecting the quality of life (QoL) and the life expectancy [3].

Bariatric surgery is beneficial in the treatment of obesity and the only one able to maintain long-term weight loss with consequent improvement or remission of obesity-related comorbidities [4].

Laparoscopic sleeve gastrectomy (LSG) is a restrictive bariatric intervention, which involves removal of a portion of the fundus, corpus, and antrum, to create a tubular duct along the lesser curvature [4]. Although initially described as a component of biliopancreatic diversion 
$[5,6]$, the popularity of LSG has increased, and today it is a stand-alone bariatric procedure, with the largest worldwide use and with effective results both for weight loss and for resolving the main comorbidities associated with obesity [7]. Several advantages linked to this procedure account for its widespread use, including the absence of an intestinal bypass and the need to perform any intestinal anastomosis [8].

In western European countries and the United States of America, LSG is well known, with published studies [9] evaluating the postoperative outcomes for the medium and long terms. Not the same can be said about Eastern Europe, where fewer reports exist. Moreover, in Eastern Europe, the number of bariatric surgical centers is smaller compared to western countries.

This study aimed to report the medium and longterm postoperative outcomes of LSG from a single surgical department in Romania, analyzing the evolution of weight loss, resolution of comorbidities and increasing QoL.

\section{Methods \\ Study design}

We conducted a retrospective and observational study, based on a survey of obese patients from a university surgical department in Târgu Mureș, Romania, who underwent surgery for obesity - LSG between May 2009 and December 2016. The inclusion criteria were the embodiment of surgery for obesity: BMI $>40 \mathrm{~kg} / \mathrm{m}^{2}$ or BMI $>35 \mathrm{~kg} / \mathrm{m}^{2}$ and associated comorbidities. To find out the weight at each point of follow-up, patients were contacted by telephone. Using a questionnaire customized after BAROS (Bariatric Analysis and Reporting Outcome System) [10] and BQLI (Bariatric Quality of Life Index) [11], we additionally assessed the perception of the patients on the improvement of the QoL after bariatric surgery. The weight loss process was analyzed by the BMI change and by the percentages of excess weight loss $(\% \mathrm{EWL})$. The excess weight was calculated by extracting the ideal weight (based on a BMI of $25 \mathrm{~kg} / \mathrm{m}^{2}$ ) from the preoperative weight. The $\% \mathrm{EWL}$ was calculated according to the formula: $[100 \mathrm{x}$ (preoperative weight - current weight)/excess weight]. Also, the postoperative weight regain was monitored (kilograms regained and duration in months). Discontinuation or dose reduction of specific medication was interpreted as remission or improvement of the main comorbidities. We excluded patients who had no proper BMI for surgery, or could not be contacted during the follow-up period.

The study was approved by the Research Ethics Committee of University of Medicine, Pharmacy, Science and Technology of Târgu Mureș.

\section{Surgical technique}

The pneumoperitoneum was performed with a Veress needle and five trocars were used. The large curvature of the stomach was dissected starting at approximately $4 \mathrm{~cm}$ from the pylorus to the esogastric junction. Stomach calibration was performed using a 36-Fr bougie, and the gastric sleeve was created by sequential application of staplers. Hemostasis at the level of the staple line was performed by applying metal clips. At the end of the intervention, the staple line was checked for any leaks by introducing the methylene blue solution through a nasogastric tube. The resected stomach (Figure 1) was extracted through the left port (through the $12 \mathrm{~mm}$ trocar place) and a drainage tube was left in place for the next 48 hours.

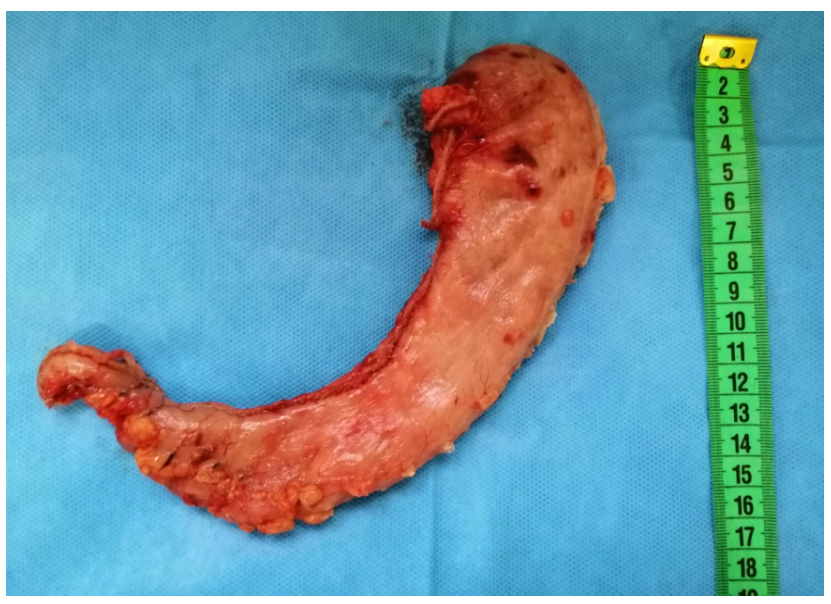

Figure 1. Specimen after laparoscopic sleeve gastrectomy.

\section{Statistical analysis}

Statistical calculations were performed using GraphPad Prism Software (San Diego, CA, USA) version 8 and Microsoft Office Excel. The quantitative data were presented as their means \pm standard deviation (SD). Qualitative variables were expressed as frequencies and percentages. Differences between preoperative and postoperative variables were determined using the ANOVA test. The tests were interpreted as being statistically significant if the value of $p$ was below the threshold value $p=0.05$.

\section{Results}

The study included 70 patients , $42(60 \%)$ of which were women, with a mean age of $47.6 \pm 9.8$ years. The main clinical characteristics of the patients are included in table I.

Table I. Characteristics of study patients.

$\begin{array}{lc}\text { Age (years), mean } \pm \text { SD } & 47.6 \pm 9.8 \\ \text { Gender (F/M), n }(\%) & 42 / 28(60 / 40) \\ \text { Weight }(\mathrm{kg}), \text { mean } \pm \mathrm{SD} & 135.3 \pm 22.5 \\ \text { BMI }\left(\mathrm{kg} / \mathrm{m}^{2}\right), \text { mean } \pm \mathrm{SD} & 47.4 \pm 6.4 \\ \text { Comorbidities, } \mathrm{n}(\%) & \\ \quad \text { Diabetes } & 15(21.4) \\ \quad \text { High blood pressure } & 47(67.1) \\ \quad \text { Snoring during sleep } & 57(81.4) \\ \text { Concomitant procedures, n }(\%) & \\ \quad \text { Cholecystectomy } & 6(8.5) \\ \quad \text { Posterior fundoplication } & 1(1.4)\end{array}$

Legend: $\mathrm{SD}=$ standard deviation, $\mathrm{BMI}=$ body mass index. 
Table II. Weight loss after laparoscopic sleeve gastrectomy.

\begin{tabular}{|c|c|c|c|c|c|c|c|c|c|c|c|}
\hline Years of follow-up & 1 & 2 & 3 & 4 & 5 & 6 & 7 & 8 & 9 & 10 & $\mathbf{p}$ \\
\hline Patients & 70 & 70 & 70 & 56 & 35 & 19 & 15 & 10 & 7 & 2 & \\
\hline \multicolumn{12}{|l|}{$\%$ EWL } \\
\hline Mean & 78.2 & 80.2 & 76.7 & 74.7 & 72.8 & 73.3 & 74.1 & 67.2 & 64.1 & 69.7 & \multirow[t]{2}{*}{$<0.0001$} \\
\hline $\mathrm{SD}$ & 20.7 & 21.5 & 21.4 & 20.8 & 22.4 & 24.6 & 20.3 & 17.2 & 20.4 & 7.7 & \\
\hline \multicolumn{12}{|l|}{ BMI } \\
\hline Mean & 30.3 & 29.9 & 30.6 & 31.1 & 31.5 & 32.1 & 31.9 & 33.6 & 34.3 & 32.8 & \multirow[t]{2}{*}{$<0.0001$} \\
\hline SD & 5.1 & 4.9 & 5.1 & 5.1 & 5.1 & 6.9 & 6.1 & 6.1 & 7.2 & 1.7 & \\
\hline
\end{tabular}

Legend: $\% \mathrm{EWL}=$ percentages of excess weight loss, $\mathrm{SD}=$ standard deviation, $\mathrm{BMI}=$ body mass index.

Preoperatively, we had 27.1\% patients with BMI over $50 \mathrm{~kg} / \mathrm{m}^{2}$ and $4.2 \%$ patients with BMI over $60 \mathrm{~kg} / \mathrm{m}^{2}$.

Concomitant procedures were performed in 6 $(8.5 \%)$ patients, most of them being cholecystectomies and in $1(1.4 \%)$ patient posterior fundoplication.

The weight loss process during follow-up, expressed as mean \%EWL and mean BMI, is shown in Table II.

Weight regain occurred in $61(87.1 \%)$ patients during the postoperative period. The mean weight regained was $8.1 \pm 4.8 \mathrm{~kg}$ at 24 months postoperatively (range 6 - 72).

$17(24.2 \%)$ patients did not have any physical activity during the postoperative period. However 22 $(30.5 \%)$ had it occasionally, and $31(44.2 \%)$ had regular physical activity.

All patients completed the questionnaire that analyzed the quality of life after LSG. Self-esteem and sexual life were the two most favorably impacted components. In addition, self-esteem had "improved" in $83 \%$ of cases. Social life and family life were "unchanged" in $64 \%$ and $71 \%$ of cases (Figure 2).

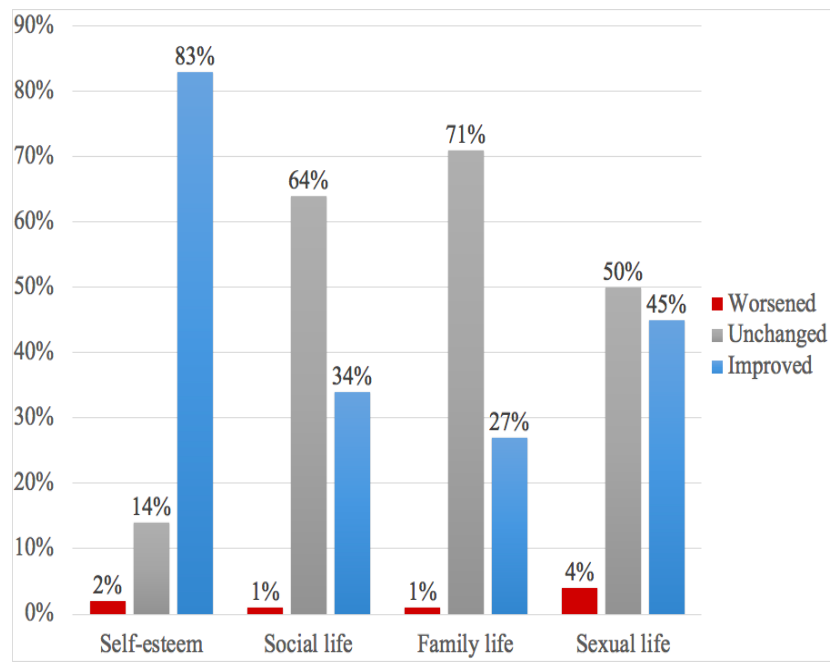

Figure 2. Quality of life after laparoscopic sleeve gastrectomy.
On a scale from 10 to 1 (10 meaning maximum) regarding the general state of physical, mental and emotional well-being, $29(41.4 \%)$ of patients chose 10 , while $3(4.2 \%)$ chose 5 . The rest of the patients were located between these values (Figure 3 ). There were no grades below 5 .

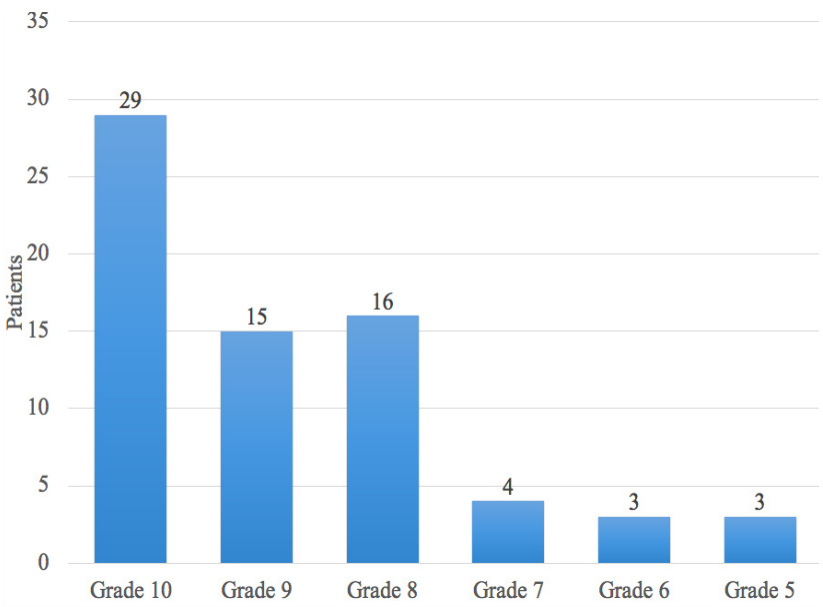

Figure 3. Scale for general state of physical, mental and emotional well-being.

Of the common obesity-related comorbidities, diabetes was found in $15(21.4 \%)$ patients, followed by high blood pressure that was found in $47(67.1 \%)$ patients; $57(81.4 \%)$ reported snoring during sleep. Table III shows the resolution of comorbidities after LSG.

Table III. Resolution of comorbidities after laparoscopic sleeve gastrectomy

\begin{tabular}{l|c|c|}
\hline Obesity-related comorbidities & Before LSG & Resolution \\
\hline Diabetes & 15 & $12(80 \%)$ \\
High blood pressure & 47 & $20(42.5 \%)$ \\
Snoring during sleep & 57 & $40(70.1 \%)$
\end{tabular}

Legend: $\mathrm{LSG}=$ laparoscopic sleeve gastrectomy. 


\section{Discussion}

In recent years, with the rise of the obesity epidemic, LSG has evolved as a primary bariatric procedure and is currently recognized by most authors as the most widespread method [8].There are published randomized trials $[12,13]$ which confirm that LSG is a safe and effective procedure for weight loss and resolution of major comorbidities.

However, issues such as long-term reporting of LSG results remain to be discussed. There are studies in the western countries that approach this subject $[14,15]$, but not the same can be said about the eastern part of Europe. Through this study we show, in a retrospective manner, the experience of a single surgical department from Târgu Mureș, Romania, in which we analyze the postoperative outcomes of LSG in the medium and long term.

This study supports the fact that LSG is an effective way to lose weight. The results of our study are comparable to other publications $[16,17]$. The randomized clinical trial published by Kehagias et al. [18] report a \%EWL after LSG of $72.9 \%$ at 12 months, $73.2 \%$ and $68.5 \%$ at 24 and 36 months, respectively.

The reason why a tendency for weight regain is seen remains unknown because the evolution of the gastric tube over time is not fully elucidated and the mechanism of weight regain is still unclear. Weight regain occurred in $61(87.1 \%)$ patients of our series, however, the average of the regained kilograms was only $8 \mathrm{~kg}$, after an average postoperative period of 2 years. Fahmy et al. [19] studied this mechanism and concluded that the gained kilograms are positively correlated with the distance between the pylorus and the start of the staple line. We applied the same technique to all patients and we started with the staple line approximately $4 \mathrm{~cm}$ from the pylorus. This distance could be considered a hypothesis in the mechanism of weight regain. Another hypothesis could be that patients have low compliance with regular visits postoperatively. Once these patients lose weight initially, they do not return for long-term follow-up, compared to patients in western countries. It was published that the lack of postoperative visits after bariatric surgery was associated with poor weight loss and weight regain [20]. At the same time, lack of physical activity and keeping old eating habits can harm weight maintenance. Our results show that $44.2 \%$ of patients regularly performed physical activity after surgery, while $24.2 \%$ did not. Other hypotheses, such as dilatation of the gastric tube or insufficient resection of the gastric fundus (place where the ghrelin is secreted) [21] could also be discussed. In a review by Karmali et al. [22] they concluded that there is a multifactorial cause that leads to weight regain, with surgical intervention and patient dependent factors, respectively.

Obesity is a disease not only through the risk of associated comorbidities but it is also cause of daily difficulties, leading to a decrease in QoL. Surgical treatment of obesity in addition to improving the QoL offers the individual a social, family, emotional and psychological reintegration [23,24]. Changes in selfimage and self-esteem after bariatric surgery have a positive influence on QoL [25]. In our patients, in $83 \%$ of cases, self-esteem after LSG increased. Social life and family life were "unchanged" in $64 \%$ and $71 \%$ of cases. On a scale from 10 to 1 (10 meaning maximum) regarding the general state of physical, mental and emotional wellbeing, $41.4 \%$ of the patients chose the maximum note 10 , and $4.2 \%$ chose the note 5 , the rest being between these intervals. There were no grades below 5 . These data come to support the increase in QoL after LSG and therefore success after bariatric surgery should include not only weight loss and resolution of comorbidities but also improvements in QoL.

Similar to other studies [26,27], we have also shown remission, namely improvement of comorbidities. Diabetes recovered, respectively improved in $80 \%$ of cases. Seven (46.6\%) patients gave up oral antidiabetics, and $5(33.3 \%)$ patients switched from insulin to oral antidiabetics. According to Juodeikis et al. [28] in a review that analyzes the long-term results after LSG, diabetes recovered in of $77.8 \%$ of the population studied.

In the case of high blood pressure, we showed that $42.5 \%$ of the cases showed remission, respectively improvement. Six (12.7\%) patients gave up antihypertensive medication, while 14 (29.7\%) reduced the dose. Casella et al. [29] report high blood pressure improvement in $38.8 \%$ of cases. In a review of 14 studies, Graham et al. [30] show that the data on remission and/or improvement of high blood pressure are heterogeneous, due to the fact that there is no standardization of the definitions of remission/improvement and also the existence of the small number of studies analyzing the results of hypertension after LSG.

Castagneto et al. [15] report sleep apnea remission in $72.2 \%$ of cases. In our study, the percentage was $70.1 \%$, but it was a subjective interpretation characterized by the lack of snoring during sleep.

There are several limitations to our study. The first limitation is represented by the retrospective manner of the study. A complete follow-up was effective only for 70 patients, which leads to the second limitation, represented by the relatively small number of patients enrolled and the single department characteristic of the investigation. Nevertheless, the current literature research indicates that the number of included cases may be statistically sufficient to draw conclusions [31,32]. The third one is given by the fact that the outcomes were collected by telephone. The fourth could be considered a limitation, because in our department we used only LSG as surgical technique for the treatment of obesity and because of that we could not compare our outcomes with other bariatric techniques outcomes. 


\section{Conclusions}

Following the results of the study, we can consider LSG as an effective bariatric procedure in the fight against obesity. The medium and long-term follow-up shows a tendency to weight regain after approximately 2 years. After LSG, a resolution of the comorbidities takes place, respectively the QoL improves. With this study, we contribute with additional information regarding the results of LSG in the medium and long term, in Eastern Europe, particularly in Romania, and we consider that the implementation of a national bariatric surgical program is required in our country.

\section{References}

1. GBD 2015. Obesity collaborators. Health effects of overweight and obesity in 195 countries over 25 years. N Engl J Med. 2017;377:13-27.

2. Guh DP, Zhang W, Bansback N, Amarsi Z, Birmingham CL, Anis AH. The incidence of co-morbidities related to obesity and overweight: a systematic review and meta-analysis. BMC Public Health. 2009;9:88.

3. Kolotkin RL, Andersen JR. A systematic review of reviews: exploring the relationship between obesity, weight loss and health-related quality of life. Clin Obes. 2017;7:273-289.

4. Angrisani L, Santonicola A, Iovino P, Vitiello A, Higa K, Himpens J, et al. IFSO Worldwide Survey 2016: Primary, Endoluminal, and Revisional Procedures. Obes Surg. 2018;28:3783-3794.

5. Hess DS, Hess DW. Biliopancreatic diversion with duodenal switch. Obes Surg. 1998;8:267-282.

6. Marceau P, Hould FS, Simard S, Lebel S, Bourque RA, Potvin M, et al. Biliopancreatic diversion with duodenal switch. World J Surg. 1998;22:947-954.

7. Gagner M, Hutchinson C, Rosenthal R. Fifth International Consensus Conference: current status of sleeve gastrectomy. Surg Obes Relat Dis. 2016;12:750-756.

8. Welbourn R, Hollyman M, Kinsman R, Dixon J, Liem R, Ottosson J, et al. Bariatric Surgery Worldwide: Baseline Demographic Description and One-Year Outcomes from the Fourth IFSO Global Registry Report 2018. Obes Surg. 2019;29:782-795.

9. Diamantis T, Apostolou KG, Alexandrou A, Griniatsos J, Felekouras E, Tsigris C. Review of long-term weight loss results after laparoscopic sleeve gastrectomy. Surg Obes Relat Dis. 2014;10:177-183.

10. Oria HE, Moorehead MK. Bariatric Analysis and Reporting Outcome System (BAROS). Obes Surg. 1998;8:487-499.

11. Weiner S, Sauerland S, Fein M, Blanco R, Pomhoff I, Weiner RA. The Bariatric Quality of Life (BQL) Index: a measure of well-being in obesity surgery patients. Obes Surg. 2005;15:538-545.

12. Helmiö M, Victorzon M, Ovaska J, Leivonen M, Juuti A, Jaser N, et al. SLEEVEPASS: a randomized prospective multicenter study comparing laparoscopic sleeve gastrectomy and gastric bypass in the treatment of morbid obesity: preliminary results. Surg Endosc. 2012;26:25212526.

13. Peterli R, Borbély Y, Kern B, Gass M, Peters T, Thurnheer $\mathrm{M}$, et al. Early results of the Swiss Multicentre Bypass or Sleeve Study (SM-BOSS): a prospective randomized trial comparing laparoscopic sleeve gastrectomy and Roux-en-Y gastric bypass. Ann Surg. 2013;258:690-694; discussion 695.

14. Arman GA, Himpens J, Dhaenens J, Ballet T, Vilallonga R, Leman G. Long-term (11+years) outcomes in weight, patient satisfaction, comorbidities, and gastroesophageal reflux treatment after laparoscopic sleeve gastrectomy. Surg Obes Relat Dis. 2016;12:1778-1786.

15. Castagneto Gissey L, Casella Mariolo JR, Genco A, Troisi A, Basso N, Casella G. 10-year follow-up after laparoscopic sleeve gastrectomy: Outcomes in a monocentric series. Surg Obes Relat Dis. 2018;14:1480-1487.

16. Kowalewski PK, Olszewski R, Walędziak MS, Janik MR, Kwiatkowski A, Galązka-Świderek N, et al. Long-Term Outcomes of Laparoscopic Sleeve Gastrectomy-a SingleCenter, Retrospective Study. Obes Surg. 2018;28:130-134.

17. Hoyuela C. Five-year outcomes of laparoscopic sleeve gastrectomy as a primary procedure for morbid obesity: A prospective study. World J Gastrointest Surg. 2017;9:109117.

18. Kehagias I, Karamanokos SN, Argentou M, Kalfarentzos F. Randomized clinical trial of laparoscopic Roux-en-Y gastric bypass versus laparoscopic sleeve gastrectomy for the management of patients with BMI $<50 \mathrm{~kg} / \mathrm{m} 2$. Obes Surg. 2011;21:1650-1656.

19. Fahmy MH, Sarhan MD, Osman AM, Badran A, Ayad A, Serour DK, et al. Early weight recidivism following laparoscopic sleeve gastrectomy: a prospective observational study. Obes Surg. 2016;26:2654-2660.

20. Vidal P, Ramón JM, Goday A, Parri A, Crous X, Trillo L, et al. Lack of adherence to follow-up visits after bariatric surgery: reasons and outcomes. Obes Surg. 2014;24:179183.

21. Bohdjalian A, Langer FB, Shakeri-Leidenmühler S, Gfrerer L, Ludvik B, Zacherl J, et al. Sleeve gastrectomy as sole and definitive bariatric procedure: 5 -year results for weight loss and ghrelin. Obes Surg. 2010;20:535-540.

22. Karmali S, Brar B, Shi X, Sharma AM, de Gara C, Birch DW. Weight recidivism post-bariatric surgery: a systematic review. Obes Surg. 2013;23:1922-1933.

23. Amichaud R, Donatini G, Barussaud ML, Charalambous C, Ingrand I, Faure JP. Health-related quality of life after laparoscopic sleeve gastrectomy. A multicentric experience. Minerva Chir. 2016;71:245-251.

24. Flølo TN, Tell GS, Kolotkin RL, Aasprang A, Norekvål TM, Våge V, et al. Changes in quality of life 5 years after sleeve gastrectomy: a prospective cohort study. BMJ Open. 2019;9:e31170.

25. Charalampakis V, Seretis C, Daskalakis M, Fokoloros C, Karim A, Melissas J. The effect of laparoscopic sleeve gastrectomy on quality of life: A prospective cohort study with 5-years follow-up. Surg Obes Relat Dis. 2018;14:1652- 
1658.

26. Hady HR, Dadan J, Luba M. The influence of laparoscopic sleeve gastrectomy on metabolic syndrome parameters in obese patients in own material. Obes Surg. 2012;22:13-22.

27. Rawlins L, Rawlins MP, Brown CC, Schumacher DL. Sleeve gastrectomy: 5-year outcomes of a single institution. Surg Obes Relat Dis. 2013;9:21-25.

28. Juodeikis Ž, Brimas G. Long-term results after sleeve gastrectomy: A systematic review. Surg Obes Relat Dis. 2017;13:693-699.

29. Casella G, Soricelli E, Giannotti D, Collalti M, Maselli R, Genco A, et al. Long-term results after laparoscopic sleeve gastrectomy in a large monocentric series. Surg Obes Relat Dis. 2016;12:757-762.

30. Graham C, Switzer N, Reso A, Armstrong C, Church N, Mitchell $\mathrm{P}$, et al. Sleeve gastrectomy and hypertension: a systematic review of long-term outcomes. Surg Endosc. 2019;33:3001-3007.

31. Major P, Matlok M, Pędziwiatr M, Migaczewski M, Budzyński P, Stanek M, et al. Quality of life after bariatric surgery. Obes Surg. 2015;25:1703-1710.

32. Hirth DA, Jones EL, Rothchild KB, Mitchell BC, Schoen JA. Laparoscopic sleeve gastrectomy: long-term weight loss outcomes. Surg Obes Relat Dis. 2015;11:1004-1007. 